\title{
Application of Deep Learning in Infrared Non-Destructive Testing
}

\author{
by Bardia Yousefi*, Davood Kalhor*, Rubén Usamentiaga**, Lei Lei*, Clemente Ibarra Castanedo*, \\ Xavier P.V. Maldague*
}

\begin{abstract}
*Computer vision and systems laboratory, Department of Electrical and Computer Engineering, Laval University, Quebec City (Quebec) G1V 0A6, Canada, Email:\{bardia.yousefi.1, davood.kalhor.1,lei.lei.1\}@ulaval.ca, \{lbarraC, xavier.maldague\}@gel.ulaval.ca

** Computer Science and Engineering Department, University of Oviedo, Edificios Departamentales Oeste, Despacho 1.1.35, Campus de Viesques, 33204 Gijón, Asturias (Spain), Email: rusamentiaga@uniovi.es
\end{abstract}

\begin{abstract}
Convolutional Neural Networks (CNN) is already known as strong tools in various fields particularly in image processing and computer vision. This paper aims to exploit the power of CNN for transform learning and utilizes it as an unsupervised feature extractor for analyzing defects in a steel specimen with float batten holes and Carbon Fiber Reinforced Plastic (CFRP) composite materials. A pre-trained CNN (ImageNet-VGG-f) has been used for extraction of the vectorized features along with a spectral angler mapper (SAM) to provide a score for defects presented in the image. Empirical results on two aforementioned datas ets indicate a promising performance for application of heating and cooling based active thermography with a reasonable computational cost due to uns upervised nature of the algorithm.
\end{abstract}

Keywords: Convolutional Neural Networks (CNN), Infrared Non-Destructive Testing (IR-NDT), Carbon Fiber Reinforced Plastic (CFRP) composite

\section{Introduction}

Convolutional Neural Networks (CNN) have been recentlyapplied byresearchers and scientists in manydifferent fields, including image processing, machine learning and pattern recognition. It has been shown also that CNN can perform well in Non-Destructive Testing \& Evaluation (NDT \& E) $[1,2]$. Therefore, it is a viable tool for performing NDT\&E analysis, particularly thermal and infrared image processing.

A CNN is a category of deep feedforward neural network that uses a variation of multilayer perceptrons with multiple hidden layers [3]. The hidden layers of CNN usuallycomprise of convolutional (cross-correlation) layers, pooling layers, fully connected layers and normalization layers [4-7]. The convolution layer, which makes CNNs different from other types of deep neural networks, is a main layer of CNN and consists of several adaptive filters (as kernels) with small receptive fields. The output of this layer is computed applying 2-dimension dotproductbetween the filter entries and the layer inputs. Consequently, in the learning process, these learnable filters learn to extract some features in the input that are more sensitivity in some spatial positions in the input. After that layer, there are several global or local pooling laye rs and combine the output information of one layer to a neuron in the next layer $[5,6]$. In the fully connected layer, similar to Multi-Layer Perceptron (MLP) networks, every neuron in one layer connects to the neurons in the next layer. Sharing weights in the convolution layer with other receptive fields improves the performance and memory efficiency [3]. A deep learning approach has been us ed for making a non-invasive approach for detecting chemically-treated collagenous tissue nonlinear anis otropic stress-strain responses in the microscopic images. A deep neural network designed in 6 layers and trained to analyze second harmonic generation (SHG) images in GLBP tissue at collagen networks. Their neural network divides into the several patches from second layer to fifth layer [14,15] and a supervised strategy in the sixth layer by regression or classification configuration. The unsupervised layers of this network involves convolution, Normalization, ReLu and Max pooling sub-layers which identically repeated, whereas the data processing (feature map) in each layer were not similar and these sizes change in this order $256^{\star} 256^{\star} 3,32^{\star} 32^{\star} 64,16^{\star} 16^{\star} 64$, and $7^{\star} 7^{\star} 64$ from second layer to fifth layer, respectively. The final feature vector provides an input to the last layer for performing classification/regress ion. Their proposed system directly calculates the mechanical properties of tissue [16]. Another approach which developed a deep learning method for soluble solid content (SSC) and firmness of pos tharvest Korla fragrant pear using Visible/NearInfrared hyperspectral reflectance (380-1030 nm) imaging including fully-connected neural network (FNN) and stacked auto-encoders (SAE). Their model comprises a hidden layer between encoding (SAE encoding initialization weighting) and decoding layers at the first and the last layer of networks which showed a rapid and non-destructive detecting [22]. 
Vetrivel et al. (2017) proposed a method for detecting disaster damage using deep learning (synergistic use) and 3D point cloud features originated by very high-resolution oblique airborne images, and multiple-kernel-learning ((MKL) which this approach is an association of a kernel-bas ed classifier such as SVM (similar [19]). The CNN used in this approach was a modified for transfer learning of imagenet-caffe-alex network [7] using two extra fully connected layers to the last layer afterfully-connected (FC) layers having different hidden layers (six layers) receptive fields of $11^{\star} 11^{\star} 96,5^{\star} 5^{\star} 256,3^{\star} 3^{\star} 384$, $3^{*} 3^{*} 384,3^{*} 3^{*} 256$, and $1 * 4096$ from $\mathrm{C} 1$ to $\mathrm{FCa}$, respectively [20]. A review paper on the application of deep learning in agriculture field, which usually summarized image processing and smart farming and food systems by overviewing 40 researches employing deep learning techniques [21]. Another method uses deeplearning for agricultural research was an automatic quality evaluation of fresh-cut iceberg lettuce through packaging material, which deployed CNN through minimum color distortions due to packing defects. This method involves patch-based CNN learning, histogram representation and kNN learning and QL classifier and was trained and tuned on 40 images (for eight samples for every quality level) and showed almost adequate segmentation capability [23]. A combination of deep learning and sparse representations used for an inclusive assessment in X-ray testing along with releasing a dataset com prising around 47.500 X-ray images having $32 \times 32$ pixels spatial resolution in automotive components with no-defects and defects. They have used several machine learning approaches for their evaluations particularly pre-trained ImageNet [17], VGG [13] type through taking one of the activation similar the proposed approach without direct quantitative assessment of the methods [18].

In this work, the vector feature weights in FC layer have been extracted from the FC7 layer of ImageNet VGG-F to be used as features for defect detection in infrared image streams (this approach can be used for many types of infrared imagery such as [7-12, 24-26, 30, 33], or even THZ imagery [28,29, 31, 32]). The reminder of these article is mentioned as follow. Next section describes the methodology and materials used for this project and brief overview on the infrared thermographical databases used for two types of composite materials and general arrangement of CNN used in this approach. Section 3, shows the results of this approach with a short discussion. Finally Section 4 concludes this article by discussion a general conclusion of the application of CNN for defect detection follows by future strategy to use CNN.

\section{Method and Material}

\section{a. Applying CNN for IRNDT}

In this paper, a pre-trained very deep convolutional network for large-scale image recognition (imagenet-vgg-verydeep-16) and ImageNet-vgg-f[13] was used. The general application of the deep learning involves heavyload of computational load due to the massive structure of the deep learning and multilayers hidden layer in its process. Moreover, training of the deep learning (i.e. CNN) follows the same properties of deep learning shortfall and requires big set of the infrared images from the specimen to be labelled and used for training of the network. This disadvantage of CNN undermines the training and testing manner of using this network. Denoting this fact, the proposed research paper premise is to use a pre -trained $\mathrm{CNN}$ to use as a hybrid feature generator. Analysis of the proposed approach involves analys is of the infrared image cube as an image in different time series, which is highlycomparable to spectral analys is and each sequence within the time of acquisition enters into the system as a raw input. Let $\boldsymbol{I}$ is an infrared image such that $\boldsymbol{I} \in \mathbb{R}^{\boldsymbol{n} \times \boldsymbol{m} \times \boldsymbol{k}}$ where $\boldsymbol{k}$ represents the time sequences, $\boldsymbol{I} \in\left\{\boldsymbol{I}_{1}, \boldsymbol{I}_{2}, \ldots, \boldsymbol{I}_{\boldsymbol{K}}\right\}$, and $\boldsymbol{n}$ and $\boldsymbol{m}$ are the spatial resolution of infrared image. If $\boldsymbol{x}$ is an infrared image the time $\boldsymbol{t}, \boldsymbol{x} \in \mathbb{R}^{\boldsymbol{A} \times \boldsymbol{B} \times \boldsymbol{k}}$ is a thermal reflectance in the $\boldsymbol{k}$ time. $\boldsymbol{x}$ re-scales to a squared matrix as an input to the ImageNetVGG-f, called $\boldsymbol{\psi}$. The utilization this image, $\boldsymbol{\psi}$, which needs to be coming a color image by concatenation of $\boldsymbol{\psi}(\boldsymbol{\psi} \rightarrow$ $\boldsymbol{\psi}_{r \boldsymbol{g} b}$ ) three times completes the CNN application process.

Applying ImageNet-vgg in the pre-trained form gave this opportunity of expanding data. Applying feed-forward convolution in neural network lookalikes of multiple-internal-functions as:

$$
g(\psi)=g_{L}\left(\ldots g_{2}\left(g_{1}\left(\psi ; w_{1}\right) ; w_{2}\right), w_{L}\right) . \quad g: \mathbb{R}^{A \times B \times C} \rightarrow \mathbb{R}^{A \prime \times B \prime \times C \prime} \quad \psi \mapsto \beta
$$

Every $\psi_{i}$ will be a $\mathbf{A} \times \boldsymbol{B} \times \boldsymbol{C}$ where real array of $\mathbf{A} \times \boldsymbol{B}$ pixels array span space (spatial resolution) has $\boldsymbol{C}$ channels per pixel span channels (time series). Let $\boldsymbol{g}_{\boldsymbol{L}}$ as a functions sethaving convolutional structure and applies to input $\boldsymbol{\psi}$. The regular linear convolution involves a filter bank where output is also follows the input dimensional property.

The CF7 was extracted for each datas et that contains a vector by the size 4096 that corresponds to the infrared contrast of every pixel. For spectral similaritymeasurement, a Spectral Angle Mapper (SAM) is an algorithm having $n$-dimensional geometrical space. It gets vector size spectrum type vector $\mathbf{F C}_{7}$ and calculate the dissimilarity error to the reference spectrum Refusing the equation below:

$$
\alpha=\cos ^{-1}\left[\frac{\sum_{\mathrm{i}=1}^{\mathrm{n}} \mathrm{FC}_{7 \mathrm{i}} \operatorname{Ref}_{\mathrm{i}}}{\left(\sum_{\mathrm{i}=1}^{\mathrm{n}} \mathrm{FC}_{7 \mathrm{i}}^{2}\right)^{1 / 2}\left(\sum_{\mathrm{i}=1}^{\mathrm{n}} \operatorname{Ref}_{\mathrm{i}}^{2}\right)^{1 / 2}}\right]
$$

Where $\boldsymbol{n}$ is the size of the vector which is 4096 pixels and represents defectiveness score (or error) obtained by each infrared image as an input to the proposed sysetm. 


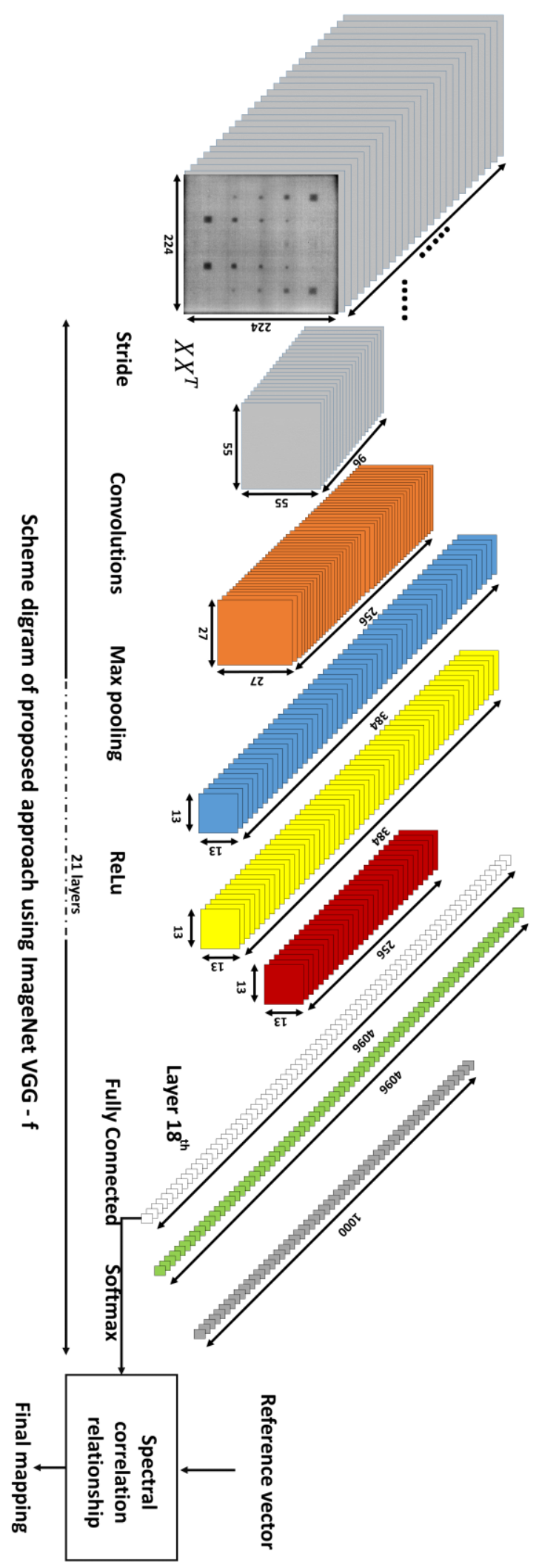

Fig. 1: the flowchart algorithm of the proposed approach is shown. The features extracted from FC7 hidden layer of ImageNet-vgg-f that it is located in $18^{\text {th }}$ layer. 


\section{Results}

The dataset used in this paper contains the heating and cooling period of active thermogra phy experiments (external heating source e.g. [8-12]) for a steel specimen with float batten holes and Carbon Fiber Reinforced Plastic (CFRP) composite material, respectively. Application of thermographyin the active form through usage of external heating source / cooling source are analyzed in this paper. The first infrared-thermography dataset used is related to CFRP composite materials and used heating source (Fig.3) in the contrast to the second dataset which involves cooling source (Fig. 2) for applying thermographic experiment. These two datasets are brieflypresented in these sections.

\section{a. Plat-bottom holes steel specimen}

In the first test, a steel specimen having flat-bottom holes of diverse sizes and depths is examined. The plate is in $10 \mathrm{~mm}$ thick, has seventeen holes at different depths, from $3 \mathrm{~mm}$ to $9 \mathrm{~mm}$, and several sizes, from $4 \mathrm{~mm}$ to $30 \mathrm{~mm}$. It was painted before the experiment to raise its emissivity and to create a homogeneous radiation source during the themal excitation. The image acquisition in this experiment is done using a FLIR SC3000 infrared camera model having $320 \times 240$ pixels spatial resolution, sampling of $50 \mathrm{~Hz}, \mathrm{GaAs}$ sensor, and sensitivity in the spectral range 8-9 $\mu \mathrm{m}$. There are two pairs of flash halogen lamps having a total of $10 \mathrm{~kJ}$ (electric) in $5 \mathrm{~ms}$ along with a bottle of liquid nitrogen and one pair of modulated halogen lamps (1KW) for Locked-in simulation [11].

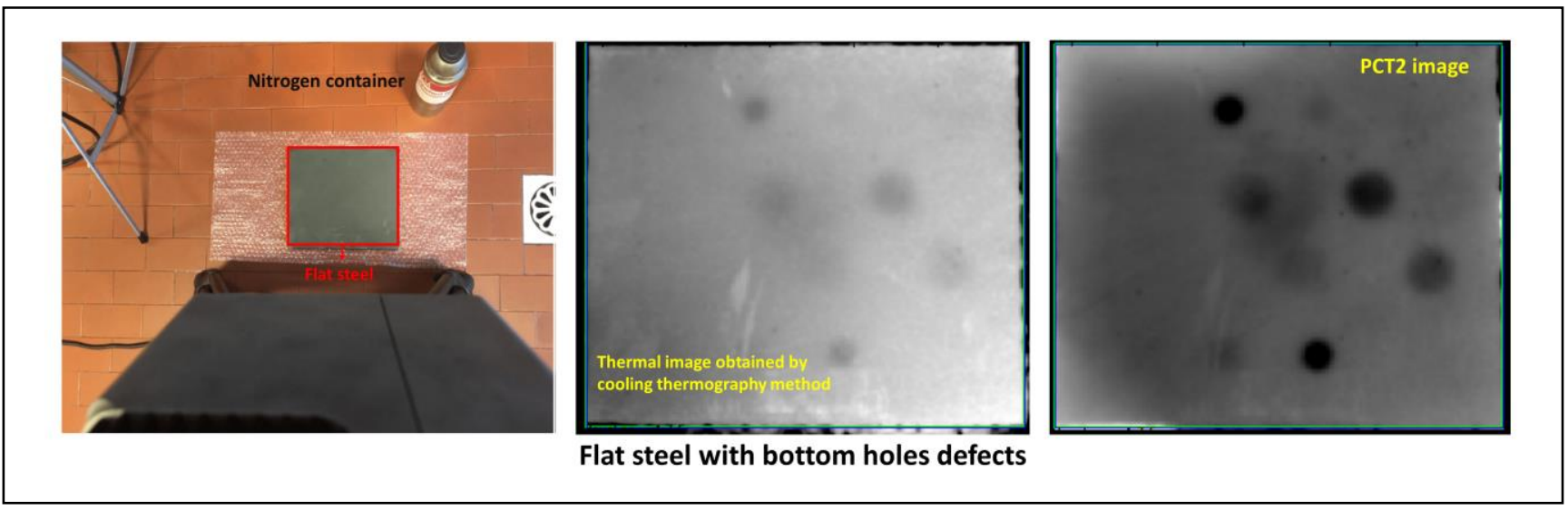

Fig. 2: Flat-bottom holes steel specimen experiment and infrared image obtained by the method are shown (pictures are adopted from [11]).

\section{b. Carbon Fiber Reinforced Plastic (CFRP) composite material specimen}

The second specimen was CFRP and its inspection was conducted from the front side of the specimen. The CFRP plate has several handmade defects created at different depth ranges, from 0.2 to $1 \mathrm{~mm}$. In the second experiment, two photographic flas hes were us ed: Balcar FX 60, $5 \mathrm{~ms}$ thermal pulse, $6.4 \mathrm{~kJ} / \mathrm{flash}$. The infrared acquisitions were performed in the Mid-Wave Infrared (MWIR), which was needed to be cooled using liquid nitrogen and had $320 \times 256$ pixels spatial resolution. In total, 1000 images were acquired from the specimen at sampling rate of $157 \mathrm{~Hz}$. 


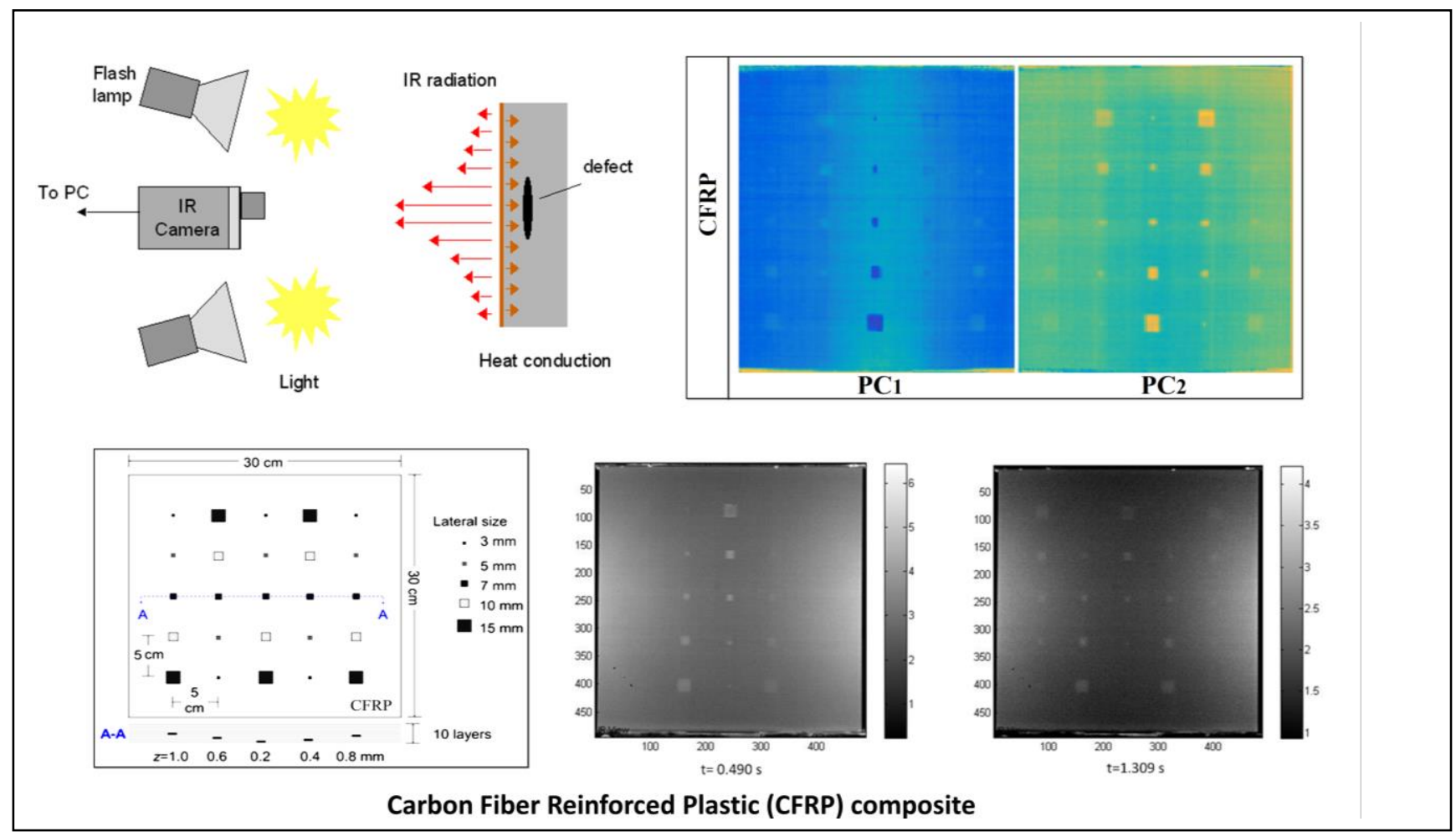

Fig. 3: Carbon Fib er Reinforced Plastic (CFRP) composite and its experimentare shown (pictures are adopted from [12]).

\section{c. Results of using FC7-CNN}

There a lot of different structures and methods to use CNN as a transfer learning or unsupervised learning approach which unequivocally involve extraction of different hidden layers and use them as features for defect determination. ImageNet-vgg-f which used for feature extraction has 21 layers with different characteristics (shown in Fig. 1) which provide various appealing size of features through breaking done the internal image input into vectors which could represent as defect determination toll. This defect assessment gave an error value throughout of the infrared sequence that gave a level of defective scene.

The proposed approach used FC7 which located in the $18^{\text {th }}$-convolution layer and contains a vector by the size of 4096 corresponds to every input image. A spectral angle extracted by SAM that could determine an error of dissimilarity between FC7 and a referencing vector. A 4096 sized vector has been used as a reference spectrum to employ SAM and gave a constant evaluation for tracking the level of defectiveness for every observation. Fig. 4 represents defect obtained by CFRP experiment and utilizing heating sources to perform active thermography. To create s uch a graph we have used four times repetition for CFRP epis ode to verify sensitivity of this criterion for amount of defects in specimens. The figure represents the level of defectiveness error are shown in every case of thermal experiment (CFRP and flat steel) with heating and cooling source application. The graph in Fig.4.a shows heating experiment using CFRP which repeated four times while the CFRP thermal images recorded episodes were ended to 200 thermal frames. Fig.4.b depicts the defectiveness level of CFRP experiment but the initial frames of thermal sequence repeated, those frames were not revealing any defects whose could notbe detected by eyes but as it is shown in the graph, the defectiveness error could catch them. Fig.4.c presents defectiveness error for flat steel with bottom holes defects experiment which employed cooling method for performing active thermography and as it is shown defects appear while the specimen was still cold (unlike heating active thermography). The graph represents the presence of defects in thermal image but with different behavior compare to heating active thermographysince defects were shown in the end of the epis ode of CFRP but for the flat steel the defect are presented in the image at the initial frames and no defect was shown at the end of cooling experiment. Consequently, the level of defectiveness at the heating experiment is ended by highest error in contrast of cooling experiment were the error is ended by lowest amount which proved the strength of the approach to determine the amount of defectiveness. 
10.21611/qirt.2018.p27

a

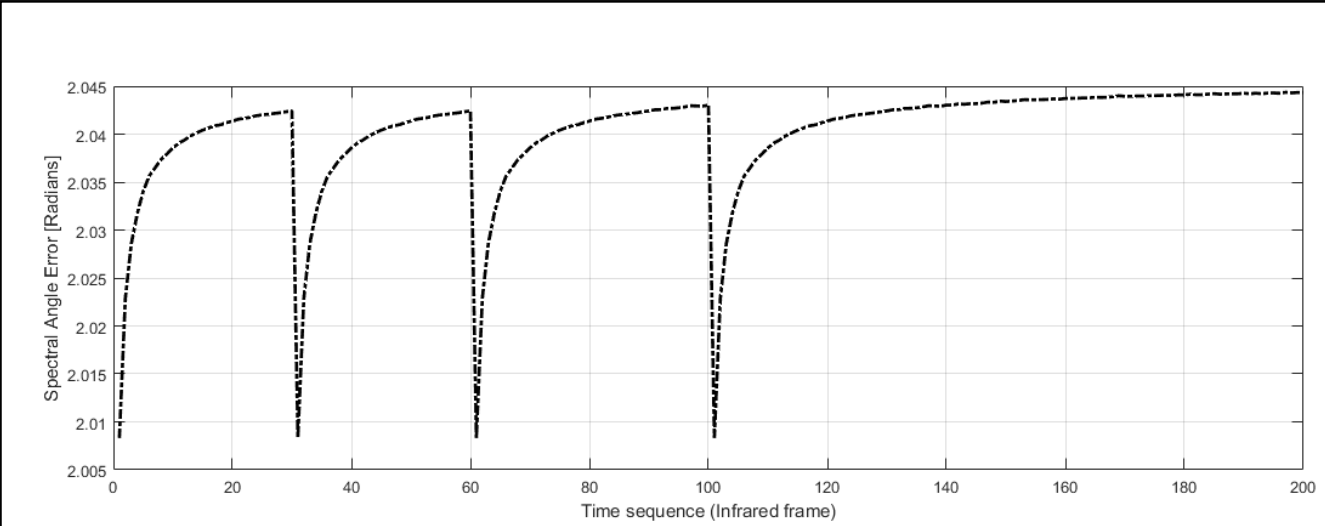

b

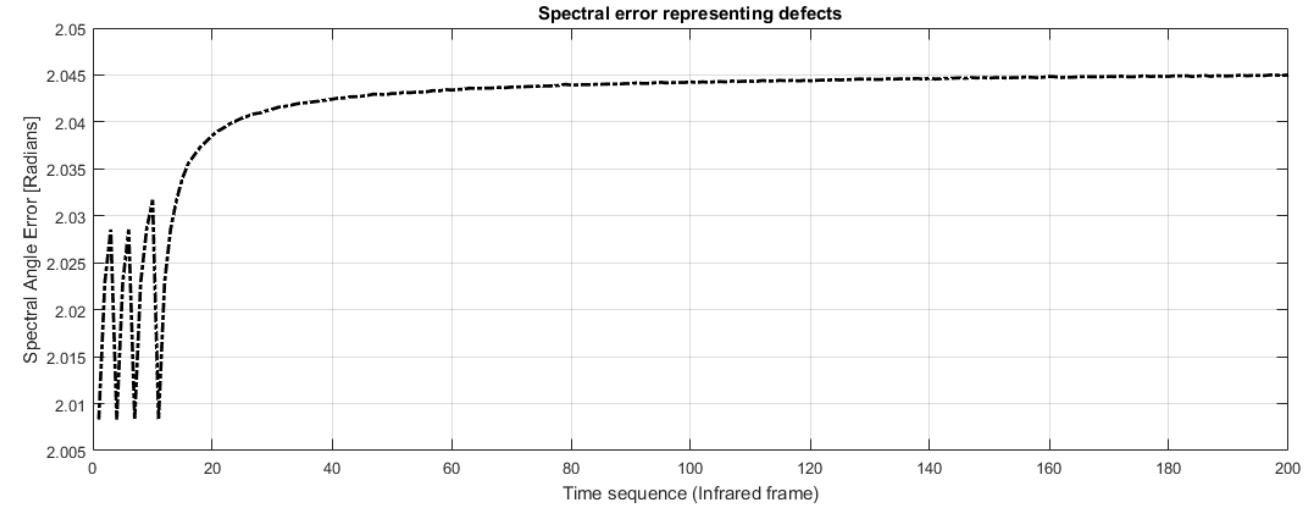

C

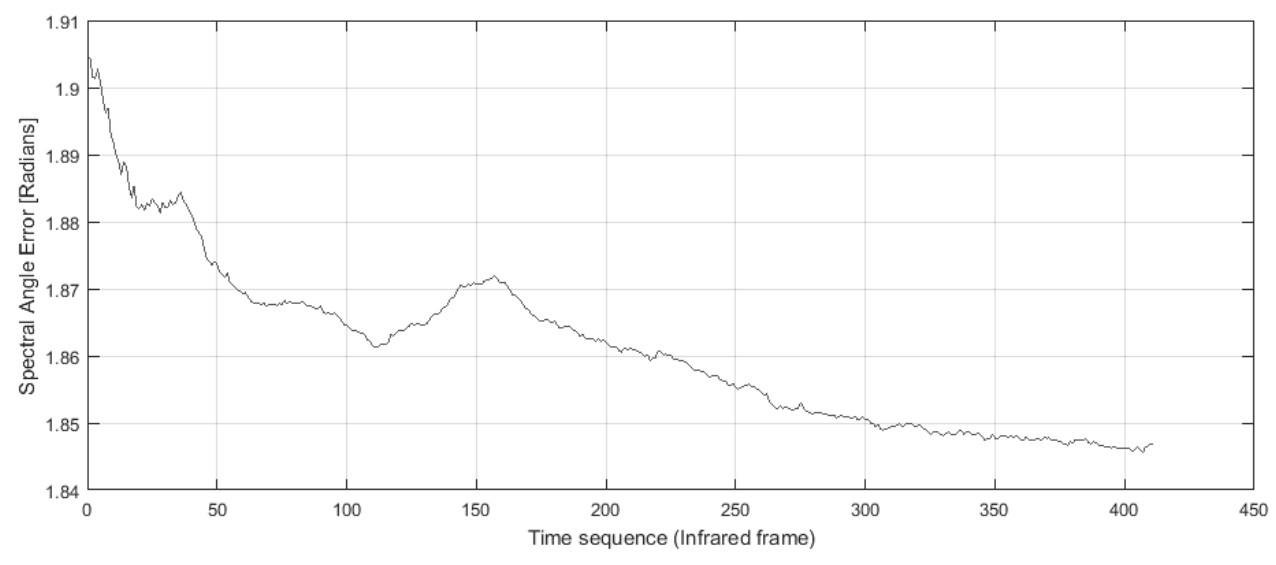

Fig. 4: The defectiveness error are shown in every case of thermal experiment (CFRP and flat steel). (a) shows four times repetition of CFRP thermal recorded episodes up to 200 thermal frames; (b) depicts the CFRP experiment but initial frames of thermal sequence repeated, those frames were not revealing any defects whose could not be detected by eyes; (c) the defectiveness error for flat steel experiment is shown applying cooling source, where defects appear while the specimen was still cold (unlike heating active thermography). . 


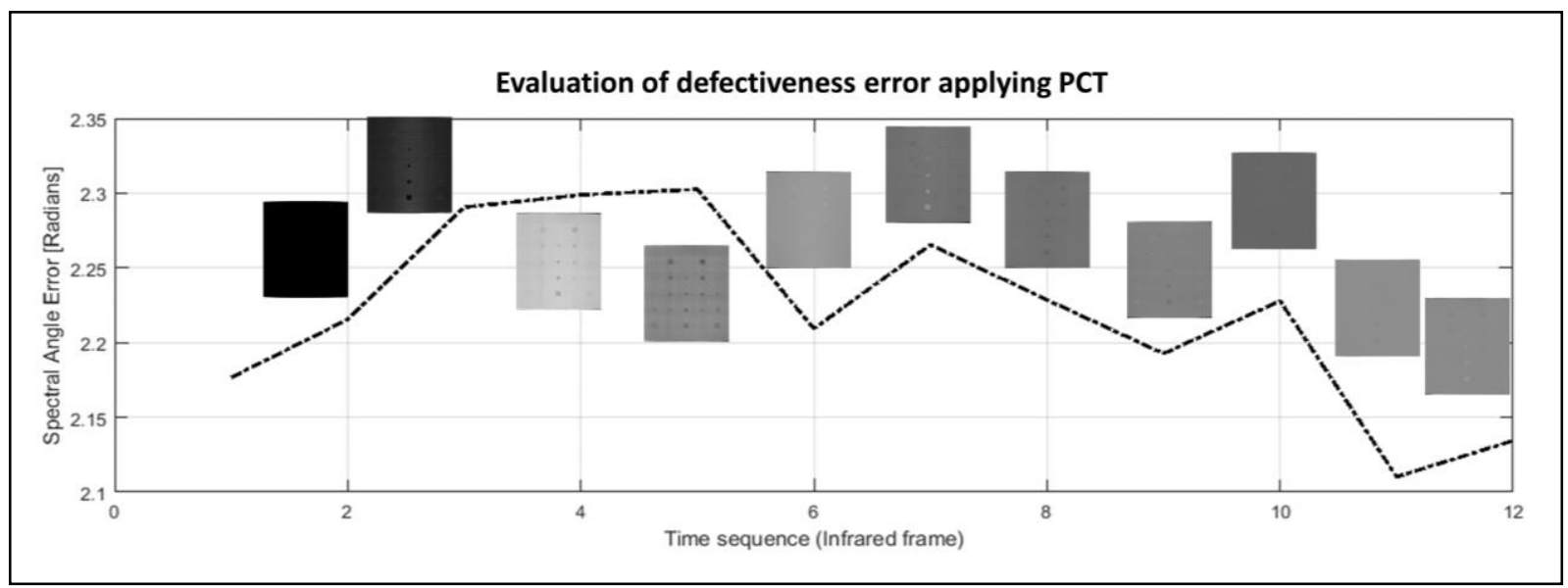

Fig.5 : The application of PCT for CFRP composite and the results of defectiveness error is showing based on the proposed approach for 12 eigen-images.

\section{d. Application for automatic rank matrix determination}

One of the important application of the proposed approach is to determine the better defect-representative eigenimages obtained by Candid Covariance-Free Incremental Principal Component Thermography (CCIPCT) [24,25], Principal Component Thermography (PCT) [26], Non-negative Matrix Factorization (NMF) [27], etc. Eigen-image establishment is one of an important concept in using decomposition method and following this intriguing necessity fi nding the better defect representative images were required an expert manual interference. The proposed approach provided a criterion that can be used as an automated determination of such methods. For that a larger set of eigen-images obtained by PCT were collected and then the approach method applied to them to find out which one has more amount of defect through evaluation of defectiveness error. Fig.5 shows an application of the proposed approach for finding the defect representative images applying PCT. As it is shown in the graph, the score increases for higher defects presents in the eigen-images and ultimately higher shows the better eigen-images.

\section{e. Computational cost}

The computational cost of the approach is mainly due to the application of ImageNet-vgg-f as every time, the algorithm needs to calculate 18 layers of convolution, max-pooling, normalization, and ReLu. However, the advantage of this approach is related to the same size input image for every time of application that is determined $224^{\star} 224$ for using ImageNet-vgg-f. The proposed algorithm ran in a PC (Intel(R) Core(TM) i7 CPU, 930, 2.2.80GHz, RAM 24.00GB, 64 bit Operating System) and the proces sing was conducted us ing MATLAB computer program. An un-optimized implementation of this method took 0.202 second for extracting the feature vector from hidden layer (FC7) and 0.02 second for employing SAM which considerable low as compare to other application of CNN. This computational efficiency could be related to using ImageNet-vgg-f which is a fats CNN-network.

\section{Conclusions}

This approach presented a Convolutional Neural Networks (CNN- ImageNet-vgg-f) for transform learning and utilized it as an unsupervised feature extractor for analyzing defects in a steel specimen with float batten holes an d Carbon Fiber Reinforced Plastic (CFRP) composite materials which had two types of active thermography using flash light heating source and liquid nitrogen as cooling source. A pre-trained CNN (ImageNet-VGG-f) has been used for extraction of the vectorized features along with a spectral angler mapper (SAM) to provide a score for defects. Empirical results on two aforementioned datasets were indicated a promising performance for application of heating and cooling based active thermography with a reasonable computational cost due to unsupervised nature of the algorithm. This research can be considered as a great opportunity to involve infrared NDT\&E image processing with deep learning concept. Possible future works could involve training of deep learning network (instead of using pre-trained network) to achieve direct defect segmentation. 


\section{Acknowledgement}

This research is conducted under a collaborative research between tier one - Multipolar Infrared Vision Canada Research Chair (MIVIM) at Department of Electrical and Computer Engineering at Laval University and Computer Science and Engineering Department, University of Oviedo.

\section{REFERENCES}

[1] Tong, Z., Gao, J., \& Zhang, H. (2017). Recognition, location, measurement, and 3D reconstruction of concealed cracks using convolutional neural networks. Construction and Building Materials, 146, 775-787.

[2] LONG, Z. J., XING, B. A., Hai, L. I. U., \& LIU, Q. H. (2017). Hyperbola Recognition from Ground Penetrating Radar Using Deep Convolutional Neural Networks. DEStech Transactions on Computer Science and Engineering, (aita).

[3] LeCun, Y. (2015). LeNet-5, convolutional neural networks. URL:http://yann. lecun. com/exdb/len et.

[4] Aghdam, H. H., \& Heravi, E. J. (2017). Guide to Convolutional Neural Networks: A Practical Application to Traffic-Sign Detection and Classification. Springer.

[5] "Convolutional Neural Networks (LeNet) - DeepLearning 0.1 documentation". DeepLearning 0.1. LISA Lab. Retrieved 31 August 2013.

[6] Ciresan, D. C., Meier, U., Masci, J., Maria Gambardella, L., \& Schmidhuber, J. (2011, July). Flexible, high performance convolutional neural networks for image classification. In IJCAI Proceedings -International Joint Conference on Artificial Intelligence (Vol. 22, No. 1, p. 1237).

[7] Krizhevsky, A., Sutskever, I., \& Hinton, G. E. (2012). Imagenet classification with deep convolutional neural networks. In Advances in neural information processing systems (pp. 1097-1105).

[8] Zhang, H., Sfarra, S., Saluja, K., Peeters, J., Fleuret, J., Duan, Y., ... \& Maldague, X. (2017). Non-destructive investigation of paintings on canvas by continuous wave terahertz imaging and flash thermography. Journal of Nondestructive Evaluation, 36(2), 34.

[9] Zhang, H., Yu, L., Hassler, U., Fernandes, H., Genest, M., Robitaille, F., ... \& Maldague, X. (2016). An experimental and analytical study of micro-laser line thermography on micro-sized flaws in stitched carbon fiber reinforced polymer composites. Composites Science and Technology, 126, 17-26.

[10] Maldague, X. (2001). Theory and practice of infrared technology for nondestructive testing.

[11] Lei, L., Ferrarini, G., Bortolin, A., Cadelano, G., Bison, P., \& Maldague, X. (2017, May). Liquid nitrogen cooling in IR thermographyapplied to steel specimen. In Thermosense: Thermal Infrared Applications XXXIX (Vol. 10214, p. 102140T). International Society for Optics and Photonics.

[12] Ibarra-Castanedo, C., \& Maldague, X. P. (2004, April). Defect depth retrieval from pulsed phase thermographic data on plexiglas and aluminum samples. In Thermosense XXVI (Vol. 5405, pp. 348-357). International Society for Optics and Photonics.

[13] Simonyan, K., \& Zisserman, A. (2014). Very deep convolutional networks for large-scale image recognition. arXiv preprint arXiv:1409.1556.

[14] Jarrett, K., Kavukcuoglu, K., \& LeCun, Y. (2009, September). What is the bestmulti-stage architecture for object recognition?. In Computer Vision, 2009 IEEE 12th International Conference on (pp. 2146-2153). IEEE.

[15] Lei, Z., Yi, D., \& Li, S. Z. (2016). Learning stacked image descriptor for face recognition. IEEE Transactions on Circuits and Systems for Video Technology, 26(9), 1685-1696.

[16] Liang, L., Liu, M., \& Sun, W. (2017). A deep learning approach to estimate chemically-treated collagenous tissue nonlinear anisotropic stress-strain responses from microscopyimages. Acta biomaterialia, 63, 227-235.

[17] Szegedy, C., Liu, W., Jia, Y., Sermanet, P., Reed, S., Anguelov, D., ... \& Rabinovich, A. (2015, June). Going deeper with convolutions. Cvpr.

[18] Mery, D., \& Arteta, C. (2017, March). Automatic Defect Recognition in X-Ray Testing Using Computer Vision. In Applications of Computer Vision (WACV), 2017 IEEE Winter Conference on (pp. 1026-1035). IEEE.

[19] Gu, Y., Wang, Q., Jia, X., \& Benediktsson, J. A. (2015). A novel MKL model of integrating LiDAR data and MSI for urban area classification. IEEE transactions on geoscience and remote sensing, 53(10), 5312-5326.

[20] Vetrivel, A., Gerke, M., Kerle, N., Nex, F., \& Vosselman, G. (2017). Disaster damage detection through synergistic use of deep learning and 3D point cloud features derived from very high resolution oblique aerial images, and multiple-kernel-learning. ISPRS journal of photogram metryand rem ote sensing.

[21] Kamilaris, A., \& Prenafeta-Boldú, F. X. (2018). Deep learning in agriculture: A survey. Computers and Electronics in Agriculture, 147,70-90.

[22] Yua X, Lua H, Wu D (2018) Development of deep learning method for predicting firmness and soluble solid content of post harvest Korla fragrant pear using Vis/NIR hyperspectral, Pos tharvest Biologyand Technology $141(2018) 39-49$.

[23] Cavallo, D. P., Cefola, M., Pace, B., Logrieco, A. F., \& Attolico, G. (2018). Non-destructive automatic quality evaluation of fresh-cut iceberg lettuce through packaging material. Journal of Food Engineering, 223, 46-52. 
[24] Yousefi, B., Sfarra, S., Castanedo, C. I., \& Maldague,X. P. (2017). Comparative analysis on thermal nondestructive testing imageryapplying Candid Covariance-Free Incremental Principal Component Thermography (CCIPCT). Infrared Physics \& Technology, 85, 163-169.

[25] Yous efi, B., Sfarra, S., Castanedo, C. I., \& Maldague, X. P. (2017, May). Thermal ndt applying candid covariance-free incremental principal component thermography (ccipct). In Thermosense:Thermal Infrared Applications XXXIX (Vol. 10214, p. 102141I). International Society for Optics and Photonics.

[26] Rajic, N. (2002). Principal component thermography for flaw contrast enhancement and flaw depth characterisation in composite structures. Composite Structures, 58(4), 521-528.

[27] Marinetti, S., Finesso, L., \& Marsilio, E. (2006). Matrix factorization methods: Application to thermal NDT/E. NDT \& E International, 39(8), 611-616.

[28] Ahi, K. (2017). Mathematical modeling of THz point spread function and simulation of THz imaging systems. IEEE Transactions on Terahertz Science and Technology, 7(6), 747-754.

[29] Ahi, K., Shahbazmohamadi, S., \& Asadizanjani, N. (2017). Quality control and authentication of packaged integrated circuits using enhanced-spatial-resolution terahertz time-domain spectroscopyand imaging. Optics and Lasers in Engineering.

[30] Usamentiaga, R., Molleda, J., Garcia, D. F., Bulnes, F. G., Entrialgo, J., \& Alvarez, C. M. S. (2015). Flatness measurement using two laser stripes to remove the effects of vibrations. IEEE Transactions on Industry Applications, 51(5), 4297-4304.

[31] Ahi, K., Lithography, Spectroscopy, and Super Resolution Terahertz Imaging for Quality Assurance and Authentication, PhD thesis, University of Connecticut (2017).

[32] Ahi, K., "Review of gan-based devices for terahertz operation," Optical Engineering 56(9), 090901 (2017).

[33] Fernandes, H., Zhang, H., Figueiredo, A., Malheiros, F., Ignacio, L. H., Sfarra, S., ... \& Maldague, X. (2018). Machine Learning and Infrared Thermography for Fiber Orientation Ass ess ment on Randomly-Oriented Strands Parts. Sensors, 18(1), 288. 